\title{
On the precision of mode delays derivation with the use of the WKB method
}

\author{
L. Maksymiuk ${ }^{1}$ - G. Stepniak ${ }^{1}$
}

Received: 3 July 2015/ Accepted: 22 August 2015/Published online: 17 December 2015

(C) The Author(s) 2015. This article is published with open access at Springerlink.com

\begin{abstract}
This paper is devoted to the problem of the WKB method inaccuracy. By comparing the WKB with a reference finite-element numerical method we show the magnitude of the WKB solution error. Moreover, we show in the paper what kind of simplifications made in the WKB analytical approximation led to its inaccuracy.
\end{abstract}

Keywords Fiber optics - Optical communications - Multimode fiber - WKB method

\section{Introduction}

In recent years one observes a revival of interest in multimode fibers. There are several key domains in which they are an interesting alternative to their single mode counterparts. These are short-range applications like: optical data interconnects (Hoffmann et al. 2011; Bigot et al. 2015), local area networks (Guillory et al. 2013) and specific radio-over-fiber designs (Maksymiuk et al. 2014). Furthermore, the fiber manufacture process has become so precise nowadays that it is technically possible to design a fiber exhibiting equal mode delays of all propagating modes. However, due to the so called profile dispersion, this is possible only in a very narrow wavelength range. In addition, several studies indicate (Qiu et al. 2013; Molin et al. 2011) that in fibers of the latest generation (like OM4) it is the chromatic dispersion that becomes the dominating source of intersymbol interference, as opposed to modal dispersion, which was regarded as the dominating distortion source for many years.

L. Maksymiuk

maksymiuk@tele.pw.edu.pl

G. Stepniak

stepniak@tele.pw.edu.pl

1 Institute of Telecommunications, Warsaw University of Technology, ul. Nowowiejska 15/19, 00-665 Warsaw, Poland 
Consequently, precise numerical studies are required in order to find optimal solutions for multimode fibers future designs in terms of minimizing modal dispersion but also in terms of fighting chromatic dispersion influence. Regardless of the particular goal, precise numerical modeling of multimode fibers requires precise input data, i.e. mode delays. Among different mathematical methods used for obtaining mode delays, the WKB (Wentzel-Kramer-Brillouin) is one of the most popular. Although the method was proposed a long time ago (Gloge and Marcatili 1973; Olshansky and Keck 1976) it is still cited and referred to by many modern books and papers in its canonical form (Matthijsse et al. 2005; Kaminov et al. 2013). The reason for such a long-lasting popularity is primarily the elegant simplicity of the closed form approximation to the WKB proposed by Gloge and Marcatili (1973), the ease of analytical solutions of mode delays and straightforward calculations of the optimal profile at particular wavelength. Unfortunately, although the analytical simplifications used in WKB derivation of fiber group delays seem very minor, we have found that they lead to an unacceptable inaccuracy of the method. In particular, when resolving the problem of the maximal bandwidth of the fiber versus wavelength, the inaccuracy of the WKB method may lead to an error of about 100-150 nm. The latter means the real optimum wavelength optimizing the bandwidth is detuned by tens of $\mathrm{nm}$ to that calculated with the use of WKB.

In this paper, we show that the key factor which leads to high inaccuracy of WKB is the negligence of the refractive index profile (parameter) shape change with respect to the wavelength. To prove this, we compare WKB method with the finite element method (FEM) mode solver, where we artificially introduced the same simplifications as in the WKB.

\section{Theoretical background}

The WKB method resolves to the well-known approximation proposed by Gloge and Marcatili (1973). The graded index multimode fibers have refractive index profile given by:

Fig. 1 Dependence of the $\alpha$ parameter versus wavelengthresults obtained with the Sellmeier equation and optimal $\alpha$ parameter versus wavelengthWKB method

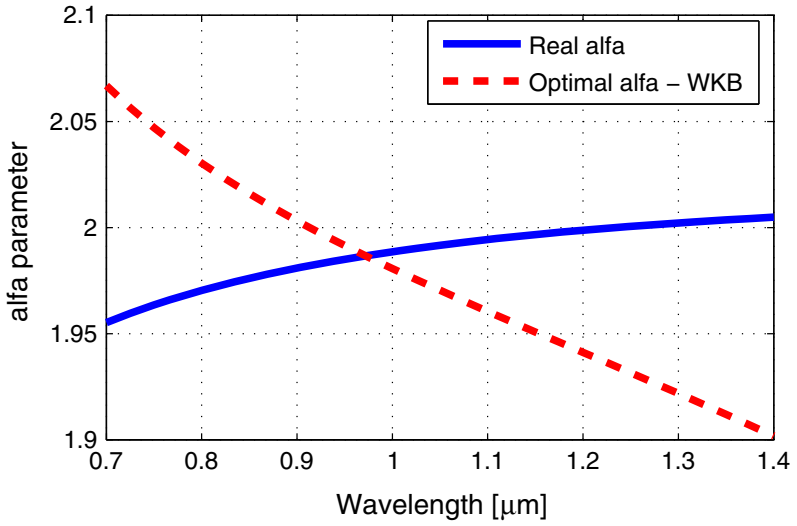




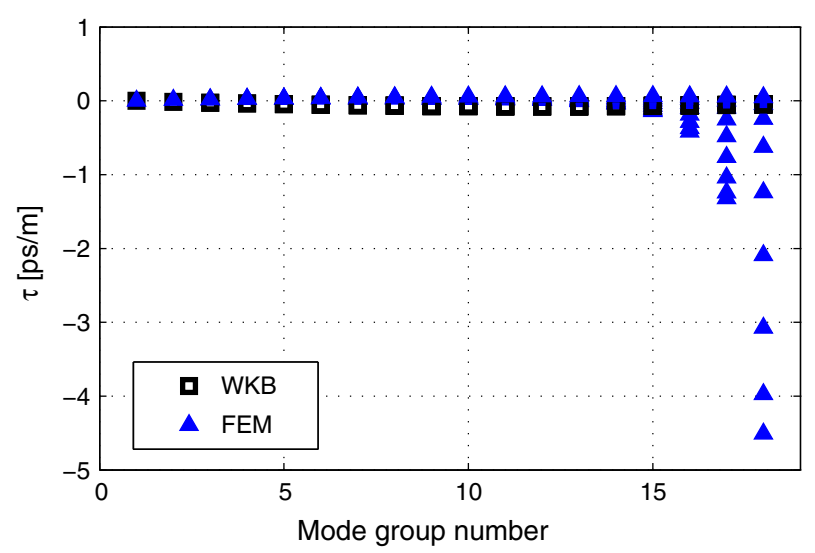

Fig. 2 Mode delays versus mode group number derived with the use of WKB and FEM methods-profile dispersion neglected, wavelength $850 \mathrm{~nm}$

$$
n(r)= \begin{cases}n_{\text {core }} \sqrt{1-2 \Delta\left(\frac{r}{a}\right)^{\alpha}} & r \leq a \\ n_{\text {core }} \sqrt{1-2 \Delta} & r>a\end{cases}
$$

where $a$ is the fiber core radius, $\alpha$ is the profile shape parameter and $\Delta$ refers to the refractive index contrast

$$
\Delta=\frac{N A^{2}}{2 n_{\text {core }}^{2}}=\frac{n_{\text {core }}^{2}-n_{\text {cladding }}^{2}}{2 n_{\text {core }}^{2}} .
$$

The propagation constants of modes are given by the following equation:

$$
\beta_{n}=n_{\text {core }} k \sqrt{1-2 \Delta\left(\frac{l}{N(\alpha)}\right)^{\frac{\alpha}{\alpha+2}}}
$$

where $k$ stands for the wave number, $l$ is the mode number and $N(\alpha)$ is the total number of modes given by:

$$
N(\alpha)=\frac{\alpha}{\alpha+2} a^{2} k^{2} n_{\text {core }}^{2} \Delta
$$

The relationship for the $l$ th mode time delay is given by the following expression (Gloge and Marcatili 1973; Olshansky and Keck 1976):

$$
\begin{aligned}
\tau_{l}=\frac{d \beta}{d \omega} & =\frac{N_{\text {core }}}{c}\left\{1+\Delta\left(\frac{\alpha-2-\epsilon}{\alpha+2}\right)\left(\frac{l}{N(\alpha)}\right)^{\frac{\alpha}{\alpha+2}}\right. \\
& \left.+\frac{\Delta^{2}}{2} \frac{3 \alpha-2-2 \epsilon}{\alpha+2}\left(\frac{l}{N(\alpha)}\right)^{\frac{2 \alpha}{\alpha+2}}\right\}+O\left(\Delta^{3}\right)
\end{aligned}
$$

where $c$ is a speed of light in vacuum and $\epsilon$ is the so called profile dispersion parameter described with the following equation: 


$$
\epsilon=\frac{-2 n_{\text {core }} \cdot \lambda}{N_{\text {core }} \Delta} \frac{d \Delta}{d \lambda} .
$$

The profile dispersion is in fact caused by the material dispersion and results in changes of the refractive index profile with wavelength. By zeroing $\epsilon$ in the Eq. (5) we may neglect the profile dispersion in the WKB. In Eqs. (5) and (6) $N_{\text {core }}$ is the group index of the material in the core center:

$$
N_{\text {core }}=n_{\text {core }}-\lambda \frac{d n_{\text {core }}}{d \lambda} .
$$

The profile dispersion parameter gives information about the optimal $\alpha$ profile that leads to the minimal pulse spread at a particular wavelength. By assuming that all the modes have equal powers, optimal $\alpha$ is given by (Olshansky and Keck 1976):

$$
\alpha_{c}=2+\epsilon-\Delta \frac{(4+\epsilon)(3+\epsilon)}{(5+2 \epsilon)} .
$$

In fact, in all the Eqs. (1)-(5) the refractive index $n$ depends on the wavelength of light. For the calculation of the wavelength dependent refractive index we apply the three term Sellmeier equation (Matthijsse et al. 2005):

$$
n^{2}-1=\sum_{i=1}^{3} \frac{a_{i} \lambda^{2}}{\lambda^{2}-\lambda_{i}^{2}} .
$$

By assuming that the fiber is pure silica glass $\left(\mathrm{SiO}_{2}\right)$ doped only with $\mathrm{Ge}$, we may use the fallowing relations for the Sellmeier coefficients (Matthijsse et al. 2005):

$$
\begin{aligned}
& a_{i}\left(X^{G e}\right)=a_{i}^{0}+X^{G e} d a_{i}^{G e} \\
& \lambda_{i}\left(X^{G e}\right)=\lambda_{i}^{0}+X^{G e} d \lambda_{i}^{G e}
\end{aligned}
$$

In Eqs. (10) and (11) X stands for the mole fraction of $G e$ which shapes the desired refractive index profile. In the forthcoming calculations we used the Sellmeier coefficients $a_{i}^{0}, d a_{i}^{G e}, \lambda_{i}^{0}$, $d \lambda_{i}^{G e}$ from (Butov and Golant 2002). In this paper we compare results obtained with the use of the WKB method with the finite-element method (FEM) used as a reference. The detailed description of the latter method was provided by Lenahan in (1983). For the sake of this paper we shall only call the equation (48) from (Lenahan 1983) describing mode delays:

$$
\tau=\sum_{l=1}^{L}\left(n^{2}\left(r_{l}\right)-\lambda^{2} \frac{d n^{2}\left(r_{l}\right)}{d \lambda^{2}}\right) \frac{g_{l}^{2}}{c n_{e}},
$$

where $n_{e}$ is the effective mode refractive index and $g$ is the vector of the mode radial field calculated numerically. The second term in the bracket is responsible for the profile dispersion, and neglecting it is like neglecting $\epsilon$ in case of the WKB method.

\section{Results and discussion}

In the series of numerical calculations presented in this paper we assumed the fiber to be a graded index pure silica glass fiber. The core refractive index was standard $50 \mu \mathrm{m}$ and 

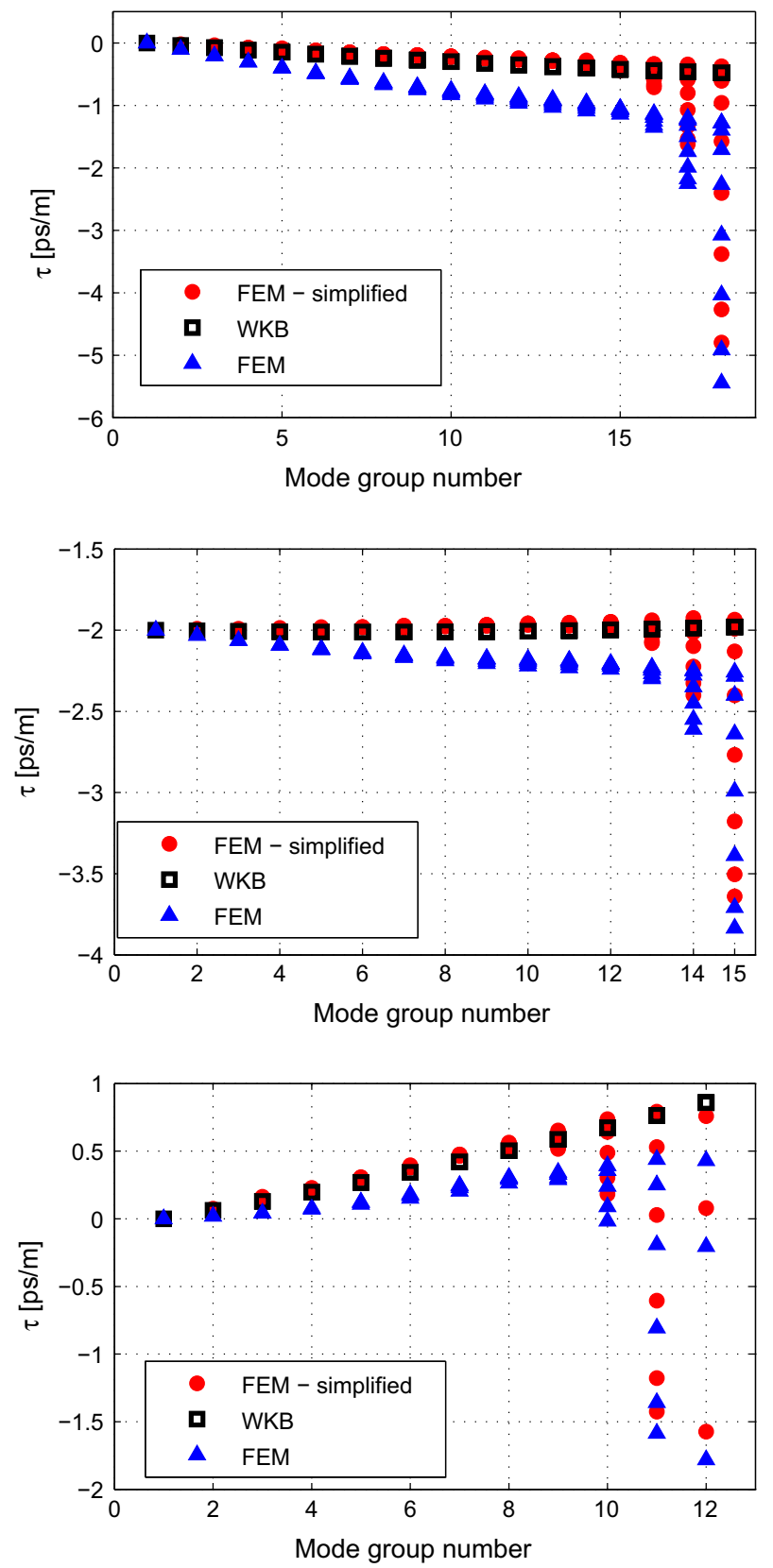

Fig. 3 Mode delays versus mode group number with respect to wavelength derived with the use of WKB, FEM and simplified FEM neglecting changes of $\alpha$ profile parameter; plots from top to bottom correspond to cases at 850, 1000 and $1300 \mathrm{~nm}$ wavelengths, respectively

numerical aperture NA $=0.2$. The dopant responsible for the profile of refraction shaping was exclusively Germanium. We used Sellmeier coefficients presented in (Butov and Golant 2002). The Germanium profile was derived to obtain the parameter 1.998 at $1 \mu \mathrm{m}$ 
Fig. 4 Dependence of the $3 \mathrm{~dB}$ modal bandwidth with respect to wavelength; results obtained with two methods: WKB and FEM

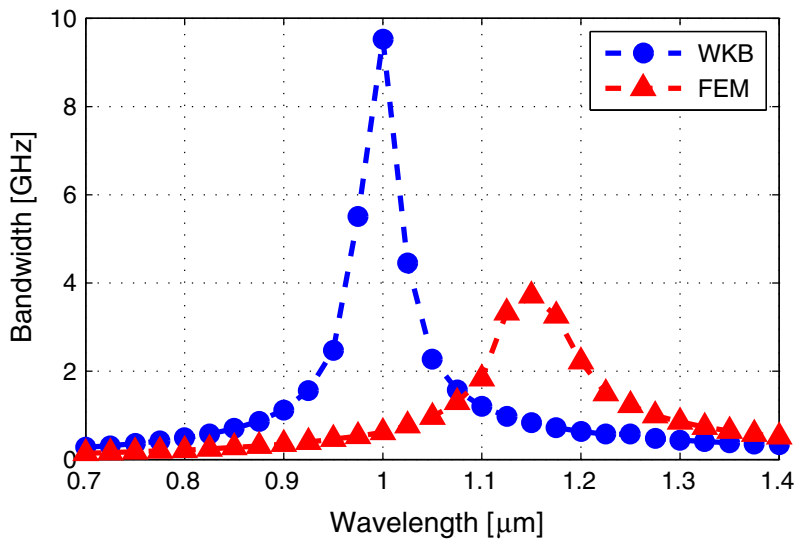

wavelength. The plot of the parameter versus the wavelength obtained with the use of the Sellmeier equation is presented in Fig. 1 together with the the optimum parameter calculated with the use of Eq. (8) the WKB method.

When we have compared the results obtained with the WKB and FEM with the profile dispersion neglected, we observed fairly good agreement between those two methods (see Fig. 2), except for the non-degenerate group delays for modes of higher order calculated with the FEM method, which contrary to the WKB takes the cladding into account.

On the other hand, including profile dispersion in the calculations (in both WKB and FEM) led to significant discrepancies between the mode delays calculated with these two methods, as shown in Fig. 3 for 3 different wavelengths. In Fig. 3 we clearly see the difference between the FEM (blue triangles) and the WKB (black squares). To understand the impact of this difference, let us analyze the $3 \mathrm{~dB}$ bandwidth versus wavelength for these two methods-see Fig. 4 (in the calculations all mode groups had the same power). We can clearly see that the maximal bandwidth is detuned by $150 \mathrm{~nm}$.

The difference is caused by the fact that the WKB approximation proposed by Gloge and Marcatili neglects the changes of the profile shape ( $\alpha$ parameter) with wavelength, as it assumes only the changes of the $\Delta$ with respect to wavelength. To prove our deduction, we have simplified the FEM in order to take into account only the changes of the $\Delta$ and not the $\alpha$. It was achieved by properly numerically calculating the term:

$$
\lambda\left(d n^{2} / d \lambda^{2}\right)\left(r_{l}\right)
$$

in such a way that the profile of refraction contrast $\Delta$ changed at a different wavelength but the $\alpha$ remained the same. The results achieved by the simplified FEM are depicted in Fig. 3 with red dots. Now we can clearly see that the modified FEM and WKB stay in a good agreement.

\section{Conclusions}

In this paper we have shown that, although the WKB (i.e. Gloge and Marcatili approximation of WKB) method is elegantly simplistic, it introduces significant inaccuracy in the calculations of mode delays. The latter leads to a significant inaccuracy in deriving the 
wavelength dependent bandwidth characteristics (150 nm shift obtained in our calculations!) or any other calculations requiring precise values of mode delays. The error of the method results from neglecting the changes of the refractive index profile shape ( $\alpha$ profile) with respect to wavelength, which was proven in this paper by means of numerical calculations. Although the changes of the fibers profile are very minor versus wavelength, they shall not be neglected. Obviously the simplification made by Glodge and Marcatili was applied in order to obtain a simple closed form analytical solution of the WKB. However, nowadays the advantage of using the simplified analytical solution is less appealing, as we are in possession of powerful tools for deriving numerical calculations.

Open Access This article is distributed under the terms of the Creative Commons Attribution 4.0 International License (http://creativecommons.org/licenses/by/4.0/), which permits unrestricted use, distribution, and reproduction in any medium, provided you give appropriate credit to the original author(s) and the source, provide a link to the Creative Commons license, and indicate if changes were made.

\section{References}

Bigot, M. et al.: Extra-wide-band OM4 MMF for future 1.6Tbps data communications. M2C.4, In: Optical Fiber Communication Conference 2015, Los Angeles, 22-26 March 2015

Butov, O.V., Golant, K.M., et al.: Refractive index dispersion of doped silica for fiber optics. Opt. Commun. 213, 301-308 (2002)

Gloge, D., Marcatili, E.A.J.: Multimode theory of graded-core fibers. Bell Syst. Tech. J. 9(9), 1563-1578 (1973)

Guillory, J., et al.: WDM-FDM approach for a multiservice home network. In: Optical Fiber Communication Conference and Exposition and the National Fiber Optic Engineers Conference (OFC/NFOEC), Anaheim, 17-21 March 2013

Hoffmann, W., et al.: $44 \mathrm{~Gb} / \mathrm{s}$ VCSEL for optical interconnects. In: Optical Fiber Communication Conference and Exposition (OFC/NFOEC), 2011 and the National Fiber Optic Engineers Conference, Los Angeles, 6-10 March 2011

Kaminov, I.P., Li, T., Wilner, A.E.: Optical Fiber Telecommunications VIA. Elsevier, Amsterdam (2013)

Lenahan, T.: Calculation of modes in an optical fiber using finite element method and eispack. Bell Syst. Tech. J. 62, 2663-2694 (1983)

Maksymiuk, L., Kowalczyk, M., Siuzdak, J.: Multimode fiber passive optical network for IEEE 802.11 signal distribution. J. Opt. Commun. Netw. 6(8), 743-753 (2014)

Matthijsse, P., Molin, D., Gooijer, F., Kuyt, G.: On the design of wide bandwidth window multimode fibers. In: Proceedings of 54th IWCS, pp. 332-337 (2005)

Molin, D., et al.: Chromatic Dispersion Compensated Multimode Fibers for Data Communications. Proc. ECOC, Tu.3.C.3 (2011)

Olshansky, R., Keck, D.B.: Pulse broadening in graded-index optical fibers. Appl. Opt. 15(2), $483-491$ (1976)

Qiu, L., et al.: 40GBASE-SR4 frame error rate test of chromatic dispersion compensating MMF. In: Optical Fiber Communication Conference and Exposition and the National Fiber Optic Engineers Conference (OFC/NFOEC), Anaheim, 17-21 March 2013 